\title{
RESULTADO NEONATAL ADVERSO EN LA ROTURA PREMATURA DE MEMBRANAS DE PREIÉRMINO SEGÚN EL MODO DEL PARTO
}

\author{
Alfredo Ovalle S. ${ }^{1}$, M. Angélica Martínez $T^{2}{ }^{2}$, Ariel Fuentes $G .{ }^{1}$, Elena Kakarieka W. ${ }^{3}$, Carlos \\ Aspillaga M. ${ }^{1}$, Ricardo Saavedra M. ${ }^{1}$, Sebastián Medel C. ${ }^{1}$ \\ ${ }^{1}$ Servicio y Departamento de Obstetricia, Ginecología y Neonatología, Hospital San Borja Arriarán. Facultad de Medicina, \\ Universidad de Chile. ${ }^{2}$ Programa de Microbiología, I.C.B.M. Facultad de Medicina, Universidad de Chile. ${ }^{3}$ Servicio de \\ Anatomía Patológica, Hospital San Borja Arriarán.
}

\begin{abstract}
RESUMEN
Objetivo: Evaluar el resultado neonatal adverso según modo de parto en la rotura prematura de membranas de pretérmino (RPMPT). Métodos: Participaron 135 embarazadas entre 24 y 34 semanas de gestación con diagnóstico de rotura prematura de membranas. Se excluyeron pacientes en trabajo de parto y condiciones maternas y fetales severas que pudiesen alterar el resultado perinatal. Todas las embarazadas tuvieron evaluación microbiológica del líquido amniótico y cérvicovaginal, y recibieron antibióticos, corticoesteroides y manejo expectante hasta las 35 semanas. Se definió resultado neonatal adverso (RA) compuesto, la variable que incluyó morbilidad neonatal severa, secuelas o muerte neonatal. Se definió invasión microbiana de la cavidad amniótica (IMCA) por cultivo positivo del líquido amniótico. Funisitis se diagnosticó por la presencia de leucocitos polimorfonucleares en la pared de los vasos umbilicales o gelatina de Warthon. La cesárea se realizó por indicaciones obstétricas o por urgencias. Para el análisis se usó curva ROC y chi cuadrado. Resultados: Se incluyeron 116 pacientes. Modo del parto: vaginal 50,1\% y cesárea $49,9 \%$. La IMCA fue $52,6 \%$ y el RA $17,2 \%$. El RA no dependió del modo del parto (vaginal $13,6 \%$ vs. cesárea $21,1 \%$ ). La vía del parto no influyó en el RA de los subgrupos donde este resultado fue más frecuente: $\leq 1500$ gramos de peso al nacer (vaginal 46,7\% vs cesárea 47,4\%) y $\leq 31$ semanas de gestación al parto (vaginal $35 \%$ vs cesárea $35,5 \%$ ). El RA se asoció con variables infecciosas: IMCA $24,6 \%$, IMCA por $S$ agalactiae $71,4 \%$, corioamnionitis histológica $100 \%$ y funisitis $94,4 \%$. Las 30 semanas de edad gestacional (Curva Roc) identificó al feto con mayor riesgo de resultado neonatal adverso según edad gestacional al parto. Conclusión: En la paciente con RPMPT manejada con antibióticos, corticosteroides y conducta expectante, el resultado neonatal adverso no depende del modo del parto.
\end{abstract}

\section{PALABRAS CLAVES: Cesárea, rotura prematura de membranas de pretérmino, resultado neonatal adverso, funisitis}

\section{SUMMARY}

Objective: To determine adverse neonatal outcome in patients with preterm PROM according to mode of delivery. Methods: 135 patients with preterm PROM between 24 and 34 weeks participated in this study. Exclusion criteria were labor, and additional fetal and maternal conditions that may influence perinatal outcome. Microbiologic assessment of amniotic cavity and the lower genital tract was performed. MIAC was defined as the presence of a positive amniotic fluid culture. Patients received antibiotics, steroids and were managed expectantly until 35 weeks. Cesarean section was performed if medically indicated or in urgency 
conditions. A composite variable including severe neonatal morbidity, sequelae or death was used. Funisitis was diagnosed in the presence of polymorphonuclear leukocyte infiltration into the umbilical vessel walls or Wharton jelly. Statistics were performed using ROC curve and chi square analysis. Results: 116 patients were included. Vaginal delivery occurred in $50.1 \%$ and cesarean delivery was performed in $49.9 \%$. Frequency of MIAC was $52.6 \%$. Adverse neonatal outcome (AO) was present in $17.2 \%$. AO is not associated with mode of delivery (vaginal $13.6 \%$ or cesarean section $21.1 \%$ ). Within the subgroups with birth weight less than $1500 \mathrm{~g}$ or 31 or less week gestation, AO was more frequent than good outcome, but there were no differences in the neonatal outcome between these subgroups with mode of delivery. AO was correlated with infectious variables: MIAC $25 \%, S$ agalactiae intraamniotic infection $71.4 \%$, histological chorioamnionitis $100 \%$ and funisitis $94 \%$. The cut off point of the ROC curve for gestational age and risk neonatal outcome was 30 weeks at labor. Conclusions: In patients with preterm PROM and expectant management with antibiotics and corticosteroids, adverse neonatal outcome no depends on mode of delivery.

\section{KEY WORDS: Cesarean section, preterm premature rupture of membranes, adverse neonatal outcome, funisitis}

\section{INTRODUCCIÓN}

La rotura prematura de membranas de pretérmino (RPMPT), se presenta en un tercio de los partos prematuros y antes de las 32 semanas de gestación se asocia con alta morbimortalidad perinatal $(1,2)$. Estos riesgos han sido relacionados con invasión microbiana de la cavidad amniótica (IMCA) (3-5) especialmente por Streptococcus agalactiae (6) y en menor grado con infección cervicovaginal (7). Las consecuencias de la infección intrauterina (corioamnionitis clínica, corioamnionitis histológica, funisitis y aumento de citoquinas intraamnióticas) en el niño se asocian con sepsis, bronconeumonía, enterocolitis necrotizante (3-5), con daños neurológicos (leucomalacia periventricular, hemorragia intraventricular, parálisis cerebral) y enfermedad crónica pulmonar (8-11). El pronóstico adverso del neonato se ha correlacionado particularmente con la funisitis, presente en el $61 \%$ de las embarazadas con IMCA $(12,13)$.

Con el uso de antibióticos y corticosteroides se han mejorado los resultados perinatales en pacientes con rotura de membranas de pretérmino y sin trabajo de parto. Con los antimicrobianos se ha conseguido prolongar la gestación (14), disminuir la sepsis, bronconeumonía y la ultrasonografía cerebral anormal en el neonato $(15,16)$. Los corticosteroides han demostrado ser útiles en reducir las tasas de síndrome de dificultad respiratoria, hemorragia intraventricular y muerte neonatal (17).

La cesárea es un modo de parto que se indica para mejorar los resultados perinatales con condiciones obstétricas adversas (18). La decisión del modo del parto en la paciente con RPMPT, manejada con conducta expectante, corticoides y anti- bióticos depende de las condiciones obstétricas y de las urgencias ocurridas. La cesárea se indica en las presentaciones podálica-tronco, cicatriz de cesárea previa, fracaso inducción de parto, infección ovular clínica (corioamnionitis clínica), cultivo de líquido amniótico positivo para bacterias de alta virulencia neonatal ( $S$ agalactiae), sufrimiento fetal, procidencia de cordón y desprendimiento prematuro de placenta (6). Es escasa la información en la literatura referente a si estas indicaciones y el momento de interrupción del parto son los adecuados para mejorar el resultados perinatal en este accidente del embarazo (19).

Los objetivos de este estudio fueron evaluar el resultado neonatal según el modo de nacimiento en pacientes con RPMPT y explorar los diferentes puntos de corte de la curva de valores de resultado neonatal adverso según edad gestacional al parto tendientes a optimizar la detección de los fetos con riesgo.

\section{MATERIAL Y MÉTODO}

Población estudiada. Embarazadas entre 24 y 34 semanas de gestación con el diagnóstico de rotura prematura de membranas (RPM) manejadas con conducta expectante, corticoides y antibióticos participaron en este estudio. El Comité de Ética del establecimiento aprobó la realización de este trabajo. Se obtuvo consentimiento informado de cada paciente participante.

La rotura de membranas fue clínicamente documentada visualizando pérdida evidente de líquido amniótico a través del orificio cervical con espéculo estéril y/o empleando la prueba de la nitrazina. La edad gestacional se determinó por 
historia menstrual o por ultrasonografía realizada antes de las 24 semanas en pacientes sin flujo menstrual confiable. Se tomaron muestras cervicales y vaginales para cultivo durante el examen con espéculo. Todas las pacientes tuvieron ultrasonografía obstétrica para biometría fetal y estudio microbiológico de líquido amniótico. Criterios de exclusión fueron: trabajo de parto, uso de antibióticos durante los 30 días previos a la admisión, hemorragia genital significativa, desprendimiento prematuro de placenta, anomalía congénita o muerte fetal, anomalías uterinas, sufrimiento fetal, presencia de dispositivo intrauterino, gestación múltiple, infección ovular clínica (corioamnionitis clínica), infección extrauterina incluyendo pielonefritis, enfermedad médica materna que determinase interrupción de la gestación y restricción del crecimiento fetal intrauterino $(<$ del percentil 10 para la edad gestacional) (20). La Figura 1 muestra el diagrama de flujo que describe el proceso de enrolamiento de pacientes.

Estudio microbiológico. Muestras cervicales, vaginales y de líquido amniótico se obtuvieron para estudio microbiológico. Una tórula de la muestra vaginal se usó para la preparación de la placa para la tinción de Gram y luego se colocó en medio de transporte de Stuart, destinado para la recuperación de $S$ agalactiae, de otras bacterias aeróbicas y anaeróbicas facultativas y de especies de Candida. Una segunda tórula endocervical fue introducida en medio de transporte SP (sacarosa fosfato) y utilizada para preparación del examen microscópico al fresco, cultivo de Mycoplasmas urogenitales y diagnóstico de $C$ trachomatis por amplificación de DNA (PCR) $(21,22)$. El examen microscópico al fresco se usó para recuento de leucocitos polimorfonucleares y para el diagnóstico de $T$ vaginalis (7).

La muestra de líquido amniótico, obtenida por amniocentesis transabdominal bajo guía ultrasonográfica, fue inoculada en medios tioglicolato prerreducido y en 2 SP. La muestra restante se transportó al laboratorio en una jeringa con cubierta estéril inmediatamente después de su recolección. El diagnóstico de bacterias anaerobias facultativas y de anaerobias estrictas, así como el cultivo de mycoplasmas urogenitales fueron realizados de acuerdo a métodos previamente descritos (23-26).

El diagnóstico microbiológico de vaginosis bacteriana se hizo usando la tinción de Gram del flujo vaginal según el método propuesto por Nugent y cols (27). Invasión microbiana de la cavidad amniótica fue definida por el cultivo positivo del líquido amniótico (28). Infección cérvicovaginal (ICV) con: a) vaginosis bacteriana $\circ$ b) cultivo positivo para bacteria patógena primaria o bacteria oportunista en cérvix o vagina (excepto Lactobacillus) asociado con incremento de leucocitos polimorfonucleares sobre 10 por campo al examen microscópico directo $(400 x)(7,29)$.

Manejo de las pacientes. El líquido amniótico se usó también para evaluación de la madurez pulmonar mediante el test de Clements. Todas las pacientes recibieron 2 dosis de betametasona 12 $\mathrm{mg}$ IM cada $24 \mathrm{~h}$ y los siguientes antibióticos: clindamicina $600 \mathrm{mg}$ IV cada 8 horas por 48 horas y gentamicina $4 \mathrm{mg} / \mathrm{kg} / \mathrm{día}$ IV en una dosis por 48 horas. Luego se continuó con clindamicina 300 $\mathrm{mg}$ oral cada 6 horas por 5 días y gentamicina 4 $\mathrm{mg} / \mathrm{kg} / \mathrm{día}$ intramuscular en una dosis por 5 días. La utilidad de este esquema está demostrada en publicación anterior de estos autores (16). No se usó tocolisis. Se manejaron las pacientes en forma expectante hasta las 35 semanas completas. Para la vigilancia fetal se realizó monitorización electrónica de la frecuencia cardíaca fetal, perfil biofísico fetal, hemograma, proteína $C$ reactiva y cultivos de líquido amniótico y endocervical semanales. El modo del parto se estableció de acuerdo a los siguientes criterios. La cesárea se realizó por indicaciones obstétricas: inicio espontáneo del parto con malas condiciones obstétricas (presentación alta), presentación podálica-tronco, cicatriz de cesárea previa y alcanzadas las 35 semanas por fracaso de la inducción de parto; indicaciones de urgencia (aparecidas durante la conducta expectante): infección ovular clínica (corioamnionitis clínica), cultivo de líquido amniótico positivo para $S$. agalactiae, $N$ gonorrhoeae, $H$ influenzae, $E$ coli o $F$ nucleatum, sufrimiento fetal, prolapso de cordón y desprendimiento prematuro de placenta (30). El parto vaginal se permitió en ausencia de estas condiciones. La placenta se envió a estudio anatomopatológico. Infección ovular clínica o corioamnionitis clínica fue definida según los criterios de Gibbs y cols (31). Durante el parto se usó clindamicina (600 mg IV cada 8 horas por 2 dosis) y gentamicina (160 mg intramuscular en una dosis por 2 días).

Complicaciones neonatales y manejo. Se comunicó a los neonatólogos el resultado del cultivo del líquido amniótico y el antibiótico recibido por la madre. Todos los neonatos bajo $2000 \mathrm{~g}$ recibieron ampicilina y amikacina endovenosa, luego de tomarse exámenes (hemograma, proteína $\mathrm{C}$ reactiva, hemocultivos y cultivos microbiológicos periféricos y de contenido gástrico). Los antibióticos se descontinuaron en ausencia de elementos clínicos de 
infección y con exámenes de laboratorio negativos.

Complicaciones de la primera semana de vida: Sepsis neonatal se diagnosticó sobre la base de cuadro multisistémico clínicamente compatible y exámenes de laboratorio tradicionales positivos. Bronconeumonía se diagnosticó con cuadro clínico y radiológico compatible. Síndrome de dificultad respiratoria (SDR) se definió como el cuadro respiratorio con quejido y retracción toráxico, con requerimientos de $\mathrm{O}_{2}$ aumentado $\left(\mathrm{FiO}_{2}>0,4\right)$, gases arteriales alterados y documentado con radiología compatible. Enterocolitis necrotizante se diagnosticó en presencia de distensión abdominal, intolerancia alimentaria $24 \mathrm{~h}$ o más, con clara evidencia radiológica de aire intramural, perforación, síndrome meconial o hallazgos específicos en la cirugía o autopsia. Hemorragia intraventricular fue diagnosticada por el examen ultrasonográfico de la cabeza del neonato y se consideraron como lesiones significativas los grados III y IV (severas hemorragias intraventriculares) (32). Asfixia severa se consideró cuando el neonato presentó test de Apgar de 3 puntos o menos a los 5 minutos de vida.

Complicaciones neonatales tardías: Leucomalacia periventricular se diagnosticó en presencia de lesiones quísticas ultrasonográficas dentro de la sustancia blanca periventricular o anormal persistencia de ecogenicidad periventricular. Displasia broncopulmonar se diagnosticó cuando el neonato requirió oxígeno y terapia ventilatoria a los 28 días de vida o a las 36 semanas de edad postconcepcional, o si tuvo típicos cambios radiográficos, o si tuvo alteraciones sugerentes de displasia broncopulmonar en la autopsia.

Estudio histológico placentario. Se tomaron muestras de tejido, del cordón umbilical, de la zona de mayor grosor de la zona central del disco placentario y de las membranas ovulares extraplacentarias. Se fijaron en formalina al $10 \%$ por 48 horas y se incluyeron en parafina. Se obtuvieron secciones de tejido de 3 a 5 micrones de espesor $y$ se tiñeron con hematoxilina y eosina ( $H$ \& E) $y$ ácido periódico de Schiff (PAS). El examen histopatológico fue realizado por un sólo patólogo (E.K.). Se estudiaron los hallazgos histopatológicos sugerentes de infección ascendente aguda: corioamnionitis (presencia de leucocitos polimorfonucleares que infiltran las membranas fetales, amnios y corion con o sin necrosis) y funisitis (inflamación aguda del cordón umbilical caracterizado por infiltración de leucocitos polimorfonucleares en la pared de los vasos umbilicales o gelatina de Warthon) (33-36).
Expresión de resultados. Para evaluar el resultado neonatal en pacientes con RPMPT, se midió el resultado neonatal adverso compuesto (37) que incluye morbilidad precoz (síndrome de dificultad respiratoria, asfixia severa, sepsis, bronconeumonía y hemorragia intraventricular severa), secuela (leucomalacia periventricular y displasia broncopulmonar) y muerte, según el modo del parto, en toda la serie y en los subgrupos 1500 gramos o menos de peso al nacer y 31 semanas o menos al parto. El resultado adverso se midió también en relación a frecuencia de IMCA, corioamnionitis histológica y funisitis. Se exploraron los diferentes puntos de corte de la curva de valores de resultado neonatal adverso según edad gestacional al parto para optimizar la detección de los fetos con riesgo.

Análisis estadístico de los datos. Se usó test de chi cuadrado y test exacto de Fisher en la comparación de proporciones; Test $\mathrm{t} \mathrm{o}$ test de Wilcoxon en la comparación de variables continuas y análisis de varianza según correspondiese. Para testear la distribución normal de las variables continuas se empleó el test de Kolmogorov. Se usó análisis de curva ROC (receiver operating characteristic) para establecer el punto de corte en la variable edad gestacional al parto, bajo el cual el riesgo para resultado neonatal adverso es significativo. Se consideró un valor $p<0,05$ como significativo.

\section{RESULTADOS}

Características de la población y exclusiones: 135 pacientes fueron enroladas en este estudio. Se excluyeron 19 mujeres: 7 casos con restricción del crecimiento fetal ( $<$ del percentil 10), 3 casos letales de malformación fetal (diagnóstico posterior al ingreso), 2 casos con membranas íntegras finalmente, 1 caso con síndrome de Down y restricción del crecimiento fetal, 1 caso con falla en la obtención de líquido amniótico, 1 caso con edad

\begin{tabular}{|c|}
\hline PACIENTES SELECCIONADAS $=745$ \\
\hline PACIENTES NO SELECCIONADAS $=610$ \\
\hline PACIENTES ENROLADAS $=135$ \\
\hline PACIENTES EXCLUIDAS $=19$ \\
\hline APROBARON CRITERIOS DE SELECCION $=116$ \\
\hline
\end{tabular}

Figura 1. Diagrama de flujo pacientes seleccionadas. 
Tabla I

POBLACIÓN EN ESTUDIO

\begin{tabular}{|c|c|c|c|}
\hline Variable & $\begin{array}{c}\text { Parto vaginal } \\
n=59\end{array}$ & $\begin{array}{c}\text { Parto cesárea } \\
n=57\end{array}$ & Valor $p$ \\
\hline \multicolumn{4}{|l|}{ Edad materna (años) } \\
\hline Media $( \pm \mathrm{DE})$ & $29,3(7,6)$ & $29,9(5,9)$ & \multirow[t]{2}{*}{ NS } \\
\hline Rango & $16-40$ & $18-43$ & \\
\hline \multicolumn{4}{|l|}{ Paridad } \\
\hline Nulípara & $18(30,5 \%)$ & $14(24,6 \%)$ & \multirow[t]{2}{*}{ NS } \\
\hline Multípara & $41(69,5 \%)$ & $43(75,4 \%)$ & \\
\hline \multicolumn{4}{|l|}{ Edad gestacional admisión (semanas) } \\
\hline Media ( $\pm \mathrm{DE})$ & $30,6(2,8)$ & $29,4(2,5)$ & \multirow[t]{2}{*}{$<0,05$} \\
\hline Rango & $24,0-34,0$ & $25,0-34,0$ & \\
\hline \multicolumn{4}{|l|}{ Intervalo admisión - parto (días) } \\
\hline Media $( \pm \mathrm{DE})$ & $8,6(7,4)$ & $12,4(10,4)$ & \multirow[t]{2}{*}{$<0,05$} \\
\hline Mediana (Rango) & $7,0(0-36)$ & $10,0(0-41)$ & \\
\hline \multicolumn{4}{|l|}{ Edad gestacional parto (semanas) } \\
\hline Media $( \pm \mathrm{DE})$ & $31,8(2,6)$ & $31,2(2,4)$ & \multirow[t]{2}{*}{ NS } \\
\hline Variación & $26,1-35,3$ & $26,4-34,5$ & \\
\hline Admisión a unidad cuidado intensivo & $34(57,6 \%)$ & $38(42,4 \%)$ & NS \\
\hline \multicolumn{4}{|l|}{ Peso nacimiento (gramos) } \\
\hline Media $( \pm \mathrm{DE})$ & $1903(506)$ & $1823(552)$ & \multirow[t]{2}{*}{ NS } \\
\hline Rango & $800-2820$ & $888-2970$ & \\
\hline
\end{tabular}

DE: Desvío estándar. NS: Diferencia no significativa.

gestacional < de 24 semanas corregida posteriormente, 1 caso de malformación uterina, 1 caso por dispositivo intrauterino y 1 caso con infección ovular clínica inadvertida. Después del enrolamiento se excluyó una muerte por trauma en el parto (caso con indicación de cesárea por presentación podálica y que nació por vía vaginal): recién nacido (739 g de peso) con hematoma hepático y muerte intraparto por shock.

116 pacientes cumplieron con los criterios de inclusión. 59 tuvieron el parto por vía vaginal $(50,1 \%)$ y $57(49,9 \%)$ por cesárea. 35 cesáreas se realizaron por indicación obstétrica y 22 de emergencia. La Tabla I describe las características de la población.

Veinte pacientes $(17,2 \%)$ tuvieron resultado neonatal adverso (RA) compuesto y 96 (82,8\%) tuvieron resultado neonatal bueno (RB). El resultado neonatal adverso no fue diferente en las indicaciones de cesáreas: obstétrica 14,3\% (5/35) vs urgencia $31,8 \%(7 / 22)$ ( $p>0,05)$. En todos los casos con cultivo de líquido amniótico positivo para $S$ agalactiae, $H$ influenzae, $F$ nucleatum y $N$ gonorrhoeae o con corioamnionitis clínica (8 pa- cientes) el parto se desencadenó espontáneamente antes de decidir la interrupción de la gestación.

La Tabla II describe los veinte casos con resultado neonatal adverso.

Resultado neonatal. El RA no dependió del modo del parto: vaginal 13,6\% (8/59) vs cesárea $21,1 \%(12 / 57)$. Tampoco la vía del parto influyó en el RA en los subgrupos donde este resultado fue más frecuente: 1500 gramos o menos de peso al nacer [parto vaginal 46,7\% (7/15) vs parto por cesárea 47,4\% (9/19)] y 31 semanas de gestación o menos al parto [parto vaginal $35 \%$ (7/20) vs. parto por cesárea 35,5\% (11/31)] (Tabla III). El RA fue significativamente más frecuente en los subgrupos $\leq 1500$ gramos de peso al nacer y $\leq 31$ semanas de gestación al parto, que en los grupos $>1500$ gramos de peso al nacer y >31 semanas de gestación o mas al parto (80\% [16/20] vs $20 \%$ [4/20]) $p<0,0001$ y $(90 \%$ [18/20 vs $10 \%$ [2/20]) $\mathrm{p}<0,0001$ respectivamente (Tabla IV).

Las 30 semanas constituyó el punto de corte de la Curva Roc para identificar al feto con mayor riesgo de resultado neonatal adverso según edad gestacional al parto; sensibilidad $82,5 \%$, especifi- 
Tabla II

RESULTADO NEONATAL ADVERSO

\begin{tabular}{|c|c|c|c|c|c|c|c|c|c|}
\hline & $S D R$ & $\begin{array}{l}\text { Asfixia } \\
\text { severa }\end{array}$ & HIV & Sepsis & $B N$ & $E N$ & $\begin{array}{c}\text { Secuela } \\
\text { severa }\end{array}$ & $\begin{array}{c}\text { Muerte } \\
\text { neonatal } \\
\text { compuesto }\end{array}$ & $\begin{array}{l}\text { Resultado } \\
\text { neonatal }\end{array}$ \\
\hline Caso 1 & & & & & si & & & & si \\
\hline Caso 2 & & & & & si & & & & si \\
\hline Caso 3 & & & & & si & & & Precoz & si \\
\hline Caso 4 & & & & & si & & & Tardía & si \\
\hline Caso 5 & & & & & si & si & & Precoz & si \\
\hline Caso 6 & & & & si & si & & & & si \\
\hline Caso 7 & & & & si & si & & & Precoz & si \\
\hline Caso 8 & & & & si & si & si & & Precoz & si \\
\hline Caso 9 & & si & & si & si & & & Precoz & si \\
\hline Caso 10 & & si & & & si & & & Tardía & si \\
\hline Caso 11 & & si & & & si & & & Precoz & si \\
\hline Caso 12 & si & & & & & & & & si \\
\hline Caso 13 & si & & & & si & & si & & si \\
\hline Caso 14 & si & si & si & & & & & & si \\
\hline Caso 15 & si & & & & & & & Precoz & si \\
\hline Caso 16 & si & & si & & & & & Precoz & si \\
\hline Caso 17 & & si & & & & & si & & si \\
\hline Caso 18 & & & si & si & si & & si & & si \\
\hline Caso 19 & & si & & & & & si & & si \\
\hline Caso 20 & & & & & si & & si & Precoz & si \\
\hline Total & $\begin{array}{c}5 \\
(4,3 \%)\end{array}$ & $\begin{array}{c}6 \\
(5,2 \%)\end{array}$ & $\begin{array}{c}3 \\
(2,6 \%)\end{array}$ & $\begin{array}{c}5 \\
(4,3 \%)\end{array}$ & $\begin{array}{c}14 \\
(12,1 \%)\end{array}$ & $\begin{array}{c}2 \\
(1,7 \%)\end{array}$ & $\begin{array}{c}5 \\
(4,3 \%)\end{array}$ & $\begin{array}{c}11 \\
(9,5 \%)\end{array}$ & $\begin{array}{c}20 \\
(17,2 \%)\end{array}$ \\
\hline
\end{tabular}

SDR: Síndrome de dificultad respiratoria HIV: hemorragia intraventricular BN: bronconeumonía. EN: enterocolitis necrotizante.

cidad $85 \%$, valor predictivo positivo $82,5 \%$, valor predictivo negativo $85 \%$. Área bajo la curva $88,7 \%$

Tabla III

RESULTADO NEONATAL SEGÚN EL MODO DE NACIMIENTO DE TODA LA SERIE Y AJUSTADO AL PESO Y A LA EDAD GESTACIONAL AL NACER

\begin{tabular}{|c|c|c|c|}
\hline & $\begin{array}{c}\text { Parto } \\
\text { vaginal } \\
n=59\end{array}$ & $\begin{array}{c}\text { Parto } \\
\text { cesárea } \\
n=57\end{array}$ & Valor $p$ \\
\hline \multicolumn{4}{|l|}{ Toda la serie } \\
\hline Resultado adverso & $8(13,6 \%)$ & $12(21,1 \%)$ & NS \\
\hline Resultado bueno & $51(86,4 \%)$ & $45(78,9 \%)$ & \\
\hline \multicolumn{4}{|l|}{$\begin{array}{l}\text { Peso al nacer } \\
\leq 1500 \text { gramos }\end{array}$} \\
\hline Resultado adverso & $7 / 15(46,7 \%)$ & $9 / 19(47,4 \%)$ & ) NS \\
\hline Resultado bueno & $8 / 15(47,4 \%)$ & $10 / 19(52,6 \%)$ & \\
\hline \multicolumn{4}{|c|}{$\begin{array}{l}\text { Edad gestacional al nacer } \\
\leq 31 \text { semanas }\end{array}$} \\
\hline Resultado adverso & $7 / 20(35,0 \%)$ & ) $11 / 31(35,5 \%)$ & NS \\
\hline Resultado bueno & $13 / 20(65,0 \%)$ & $20 / 31(64,5 \%)$ & \\
\hline
\end{tabular}

NS: Diferencia no significativa.
(Figura 2). En la Figura 3 se analiza el resultado neonatal según edad gestacional y peso al nacer. 17 de los veinte casos con RA tuvieron 1500 gramos o menos de peso al nacer y 30 semanas de gestación o menos al parto.

Microbiología del tracto genital inferior y del líquido amniótico. Las bacterias más frecuentemente aisladas de cérvix/vagina y del líquido am-

Tabla IV

RESULTADO NEONATAL AJUSTADO A PESO Y A EDAD GESTACIONAL AL PARTO

\begin{tabular}{|c|c|c|c|}
\hline Variable & $\begin{array}{c}\text { Resultado } \\
\text { adverso } \\
n=20\end{array}$ & $\begin{array}{c}\text { Resultado } \\
\text { bueno } \\
n=96\end{array}$ & Valor $p$ \\
\hline \multicolumn{4}{|c|}{ Peso al nacer (gramos) } \\
\hline$\leq 1500$ & $16(80,0 \%)$ & $18(18,8 \%)$ & $<0,0001$ \\
\hline$>1500$ & $4(20 \%)$ & $78(81,2 \%)$ & \\
\hline \multicolumn{4}{|c|}{$\begin{array}{l}\text { Edad gestacional al } \\
\text { parto (semanas) }\end{array}$} \\
\hline$\leq 31$ & $18(90,0 \%)$ & $33(34,4 \%)$ & \\
\hline$>31$ & $2(10,0 \%)$ & $63(65,6 \%)$ & $<0,0001$ \\
\hline
\end{tabular}




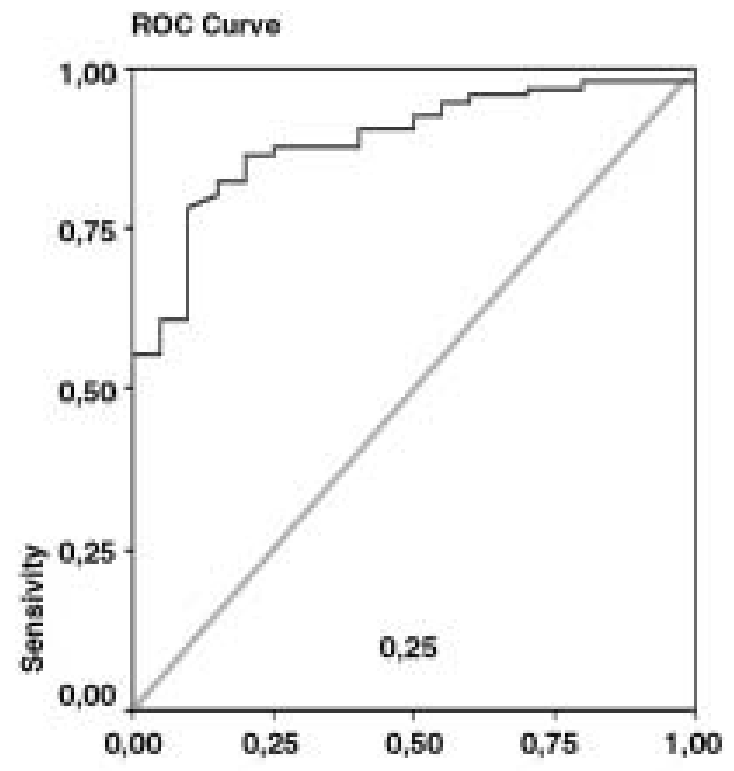

\section{1 - Specifieity}

\section{Diagonal segments are produced by ties.}

Figura 2. Análisis de Curva Roc en pacientes con resultado neonatal adverso según edad gestacional al parto. Punto de corte 30 semanas de gestación; sensibilidad $82,5 \%$, especificidad $85 \%$, valor predictivo positivo $82,5 \%$, valor predictivo negativo $85 \%$. Área bajo la curva $88,7 \%$

niótico se presentan en Tabla V. No hubo diferencias en los hallazgos microbiológicos de las pacientes atendidas por parto vaginal o por cesárea. La frecuencia de IMCA de toda la serie fue 52,6\% (61/116). Todas las pacientes con IMCA tuvieron ICV. Infección cérvicovaginal sin infección intrauterina se presentó en el 27,6\% (32/116). Pacientes sin infección (cérvix/vagina y líquido amniótico) $19,8 \%(23 / 116)$. La frecuencia de vaginosis bacteriana fue $37,1 \%(43 / 116)$.

La IMCA se asoció con significativa mayor frecuencia de RA, que la ICV sin infección intrauterina y que la ausencia de infección (cérvix/vagina y líquido amniótico): 24,6\% (15/61) vs 15,6\% (5/ 32) vs $0 \%(0 / 23) p=0,03$ respectivamente. $S$. agalactiae se aisló más frecuentemente del líquido amniótico en el grupo con RA 25,0\% (5/20), que en el grupo con RB 2,1\% (2/96), p<0,01. La IMCA por $S$ agalactiae, tuvo $71,4 \%(5 / 7)$ de RA.

Hallazgos histológicos placentarios según grupos. La frecuencia de corioamnionitis histológica y de funisitis no fue diferente en las pacientes atendidas por parto vaginal o por cesárea [64,6\% (31/

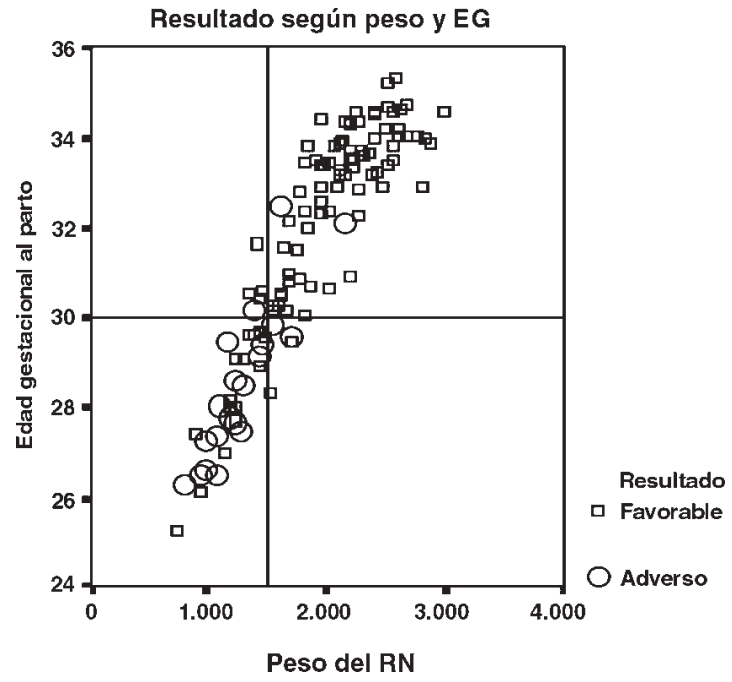

Figura 3. Resultado neonatal según edad gestacional y peso al nacer.

$48)$ vs. $63,8 \%(30 / 47)$ y $43,8 \%(21 / 48)$ vs $53,2 \%$ (25/47)] respectivamente. Tampoco el RA con corioamnionitis histológica y con funisitis fue diferente en las pacientes atendidas por parto vaginal 0 por cesárea $[22,6 \%(7 / 31)$ vs. $36,7(11 / 30)$ y $28,6 \%$ $(6 / 21)$ vs. $44,0 \%(11 / 25]$ respectivamente. Sin embargo el grupo con RA, presentó más frecuentemente corioamnionitis histológica y funisitis, que el grupo con RB (100\% [18/18] vs 55,8\% [43/77]) $p<0,001$ y $(94,4 \% \quad[17 / 18]$ vs $37,7 \%$ [29/77]) $\mathrm{p}<0,0001$ respectivamente (Tabla VI).

\section{DISCUSIÓN}

Este estudio demuestra que en la paciente con RPMPT con elevada frecuencia de IMCA (53\%), manejada con antibióticos, corticosteroides y conducta expectante, el resultado neonatal adverso no depende del modo del parto. Tampoco el RA es diferente según la vía de nacimiento en los subgrupos donde este resultado es más frecuente, 1500 gramos o menos de peso al nacer y 31 semanas de gestación o menos al parto. Este trabajo sugiere además, que la cesárea en la embarazada con RPMPT indicada por condiciones obstétricas adversas y urgencias ocurridas, no mejora el resultado perinatal.

La rotura prematura de membranas de pretérmino, antes de las 32 semanas de gestación se asocia con alta morbilidad neonatal $(1,2)$ sepsis, bronconeumonía, enterocolitis necrotizante (3-5) y 
Tabla V

\section{MICROORGANISMOS AISLADOS DE PACIENTES CON INFECCIÓN CERVICOVAGINAL E INVASIÓN MICROBIANA DE LA CAVIDAD AMNIÓTICA}

\begin{tabular}{|c|c|c|c|c|c|c|}
\hline \multirow[b]{2}{*}{ Microorganismos } & \multicolumn{2}{|c|}{$I C V$} & \multicolumn{4}{|c|}{$I M C A$} \\
\hline & $\begin{array}{c}\text { Resultado } \\
\text { adverso } n=20\end{array}$ & $\begin{array}{c}\text { Resultado } \\
\text { bueno } n=96\end{array}$ & $\begin{array}{c}\text { Valor } \\
p\end{array}$ & $\begin{array}{c}\text { Resultado } \\
\text { adverso } n=20\end{array}$ & $\begin{array}{c}\text { Resultado } \\
\text { bueno } n=96\end{array}$ & $\begin{array}{c}\text { Valor } \\
p\end{array}$ \\
\hline Ureaplasma urealyticum & $11(55,0 \%)$ & $56(58,3 \%)$ & NS & $7(35,0 \%)$ & $36(37,5 \%)$ & NS \\
\hline Gardnerella vaginalis & $11(55,0 \%)$ & $39(40,6 \%)$ & NS & $2(10,0 \%)$ & $11(11,5 \%)$ & NS \\
\hline Mycoplasma hominis & $4(20,0 \%)$ & $17(17,7 \%)$ & NS & $2(10,0 \%)$ & $6(6,3 \%)$ & NS \\
\hline Streptococcus agalactiae & $6(30,0 \%)$ & $14(14,6 \%)$ & NS & $5(25,0 \%)$ & $2(2,1 \%)$ & $<0,01$ \\
\hline Streptococcus viridans & $4(20,0 \%)$ & $10(10,4 \%)$ & NS & $1(5,0 \%)$ & $3(3,1 \%)$ & NS \\
\hline Haemophilus influenzae & $2(10,0 \%)$ & $3(3,1 \%)$ & NS & $1(5,0 \%)$ & $4(4,2 \%)$ & NS \\
\hline Enterococcus sp & $2(10,0 \%)$ & $3(3,1 \%)$ & NS & $1(5,0 \%)$ & $1(1,0 \%)$ & NS \\
\hline Fusobacterium nucleatum & 0 & 0 & & $2(10,0 \%)$ & $1(1,0 \%)$ & NS \\
\hline Prevotella sp & 0 & 0 & & $1(5,0 \%)$ & $3(3,1 \%)$ & NS \\
\hline Peptostreptococcus sp & 0 & 0 & & $1(5,0 \%)$ & $2(2,1 \%)$ & NS \\
\hline Staphylococcus coagulasa (-) & 0 & $4(4,2 \%)$ & NS & 0 & $2(2,1 \%)$ & NS \\
\hline Candida albicans & 0 & $5(5,2 \%)$ & NS & 0 & $1(1,0 \%)$ & NS \\
\hline Streptococcus pneumoniae & 0 & $1(1,0 \%)$ & NS & $1(5,0 \%)$ & $1(1,0 \%)$ & NS \\
\hline Bacteroides fragilis & 0 & 0 & & $1(5,0 \%)$ & 0 & NS \\
\hline Neisseria gonorrhoeae & 0 & $1(1,0 \%)$ & NS & 0 & $1(1,0 \%)$ & NS \\
\hline Enterobacter sp & 0 & $1(1,0 \%)$ & & 0 & $1(1,0 \%)$ & NS \\
\hline Escherichia coli & 1 & $4(4,2 \%)$ & NS & 0 & 0 & \\
\hline Chlamydia trachomatis & 0 & $2(2,1 \%)$ & NS & 0 & 0 & \\
\hline Klebsiella pneumoniae & 0 & $2(2,1 \%)$ & NS & 0 & 0 & \\
\hline Trichomona vaginalis & 0 & $2(2,1 \%)$ & NS & 0 & 0 & \\
\hline Proteus mirabilis & 0 & $1(1,0 \%)$ & NS & 0 & 0 & NS \\
\hline
\end{tabular}

ICV: infección cervicovaginal. IMCA: invasión microbiana de la cavidad amniótica.

Vaginosis bacteriana: 55,0\% (11/20) resultado adverso, 33,3\% (32/96) resultado bueno. NS: diferencia no significativa.

mortalidad por estas causas, con daños neurológicos (leucomalacia periventricular, hemorragia intraventricular, parálisis cerebral) y con enfermedad crónica pulmonar (8-11). Estos riesgos han sido directamente relacionados con corioamnionitis clínica, corioamnionitis histológica, funisitis y aumento de citoquinas intraamnióticas causados por la invasión microbiana de la cavidad amniótica (IMCA) (3-5) especialmente por $S$ agalactiae (6). Con el uso de antibióticos y corticosteroides se han mejorado los resultados perinatales en estas pacientes. Con los antimicrobianos se ha conseguido prolongar la gestación, (14) disminuir la sepsis, bronconeumonía y la ultrasonografía cerebral anormal en el neonato $(15,16)$. Con los corticosteroides se han reducido las tasas de síndrome de dificultad respiratoria, hemorragia intraventricular y muerte neonatal (17).

Sin embargo con la cesárea, modo del parto indicado para mejorar el resultado del nacimiento, de acuerdo a los resultados publicados en la literatura y en este estudio (19), en la paciente con RPMPT no se ha logrado este objetivo. La falla de la cesárea en mejorar el resultado perinatal en esta serie con alta frecuencia de IMCA (53\%) y en que se usaron antibióticos de eficacia probada, (16) se puede explicar por los siguientes argumentos infecciosos:

1. El RA, se relacionó con variables infecciosas, independientes del modo del parto: IMCA $25 \%$, especialmente por $S$ agalactiae $71 \%$, funisitis y corioamnionitis histológica existentes en el $94 \%$ y en todos los casos respectivamente.

Tabla VI

HALLAZGOS HISTOLÓGICOS PLACENTARIOS SUGERENTES DE INFECCIÓN ASCENDENTE AGUDA SEGÚN EL RESULTADO NEONATAL

\begin{tabular}{lccc}
\hline $\begin{array}{l}\text { Hallazgos } \\
\text { histológicos }\end{array}$ & $\begin{array}{c}\text { Resultado } \\
\text { adverso* } \\
n=18\end{array}$ & $\begin{array}{c}\text { Resultado } \\
\text { bueno** } \\
n=77\end{array}$ & Valor $p$ \\
\hline Corioamnionitis & $18(100 \%)$ & $43(55,8 \%)$ & $<0,001$ \\
Funisitis & $17(94,4 \%)$ & $29(37,7 \%)$ & $<0,0001$ \\
\hline
\end{tabular}

*Se excluyen 2 casos sin estudio placentario.

${ }^{* *}$ Se excluyen 19 casos sin estudio placentario. 
2. La cesárea de emergencia indicada por corioamnionitis clínica, cultivo de líquido amniótico positivo para $S$ agalactiae (u otra bacteria virulenta), es posible que se haya realizado con infección avanzada. Otras indicaciones de urgencia, sufrimiento fetal y desprendimiento prematuro de placenta, también pueden corresponder a expresiones clínicas de infección intrauterina severa, que sin duda aumentan el riesgo fetal (38).

De este modo, ni los antibióticos fueron suficientes, ni la cesárea de emergencia fue oportuna para evitar la morbimortalidad neonatal secundaria a la funisitis y al aumento de citoquinas intraamnióticas, consecuencias de la infección intrauterina $(6,39,40)$.

Estos resultados coinciden con lo sostenido en varias publicaciones, que los riesgos perinatales han sido directamente relacionados con la invasión microbiana de la cavidad amniótica y sus consecuencias (3-6).

En esta serie el punto de corte de la Curva Roc nos ha servido para conocer el feto que tiene mayor riesgo de resultado neonatal adverso según edad gestacional al parto. Este punto fue 30 semanas; sensibilidad $82,5 \%$, especificidad $85 \%$, valor predictivo positivo $82.5 \%$, valor predictivo negativo $85 \%$.

Es probable que la cesárea pueda mejorar los resultados perinatales en pacientes con RPM de PT manejadas con antibióticos, corticosteroides y conducta expectante, especialmente en el grupo de mayor riesgo (gestación menor de 30 semanas) si se anticipa el momento de interrupción del embarazo, en base a recomendaciones tendientes a disminuir el impacto de la infección sobre el feto:

1. Hacer prontamente la cesárea en pacientes con IMCA por $S$ agalactiae, $F$ nucleatum, $H$ influenzae, $E$ coli o $N$ gonorrhoeae, bacterias de reconocida virulencia para el feto (6), antes de la aparición de funisitis severa y elevación importante de la inflamación intraamniótica durante la conducta conservadora. Para esto es conveniente mejorar el diagnóstico microbiológico usando la técnica molecular por PCR (reacción de polimerasa en cadena) y usar la medición seriada de IL-6 $(8,41)$. Además es adecuado usar perfil biofísico fetal y Doppler de la arteria umbilical que permiten predecir cuando un feto está en riesgo (38).

2. Hacer cesárea inmediatamente en pacientes con IMCA y sospecha de corioamnionitis clínica, sufrimiento fetal y desprendimiento prematuro de placenta.

Nuevos estudios se requieren para determinar si en la RPM de PT, el conocimiento acabado de los riesgos que la IMCA produce en el feto, permite mediante la cesárea anticipar el momento de interrupción del embarazo y mejorar el resultado perinatal.

\section{BIBLIOGRAFÍA}

1. Meis PJ, Ernest JM, Moore ML. Causes of low birth weight births in public and private patients. Am J Obstet Gynecol 1987;156:1165-8.

2. Gibbs RS, Blanco JD. Premature rupture of the membranes. Obstet Gynecol 1982;60:671-9.

3. Romero R, Ghidini A, Mazor M, Behnke E. Microbial invasion of the amniotic cavity in premature rupture of membranes. Clin Obstet Ginecol 1991;4:769-78.

4. Romero R, Quintero R, Oyarzún E, Wu YK, Sabo V, Mazor M, et al. Intraamniotic infection and the onset of labor in preterm premature rupture of the membranes. Am J Obstet Gynecol 1988;159:661-6.

5. Gómez R, Ghezzi R, Romero R, Muñoz H, Tolosa J, Rojas Iván. Premature labor and intra-amniotic infection. Clin Perinatol 1995;22:281-342.

6. Ovalle A, Gómez R, Martínez MA, Kakarieka E, Fuentes A, Aspillaga C, et al. Invasión microbiana de la cavidad amniótica en la rotura de membranas de pretérmino. Resultados maternoneonatales y patología placentaria según microorganismo aislado. Rev Med Chile 2005;133:55-61.

7. Ovalle A, Martínez MA, Giglio MS, Poblete P, Fuentes A, Villablanca E. Microbiología aislada en la rotura prematura de membranas de pretérmino. Relación con morbilidad infecciosa materna neonatal e intervalo rotura de membranas-parto. REV CHIL Obstet GineCol 1995;60:252-62.

8. Gómez R, Romero R, Ghezzi F, Yoon BH, Mazor M, Berry SM. The fetal inflammatory response syndrome. Am J Obstet Gynecol 1998;179(1):194-202

9. Hagberg H, Wennerholm UB, Savman K. Sequelae of chorioamnionitis. Curr Opin Infect Dis 2002;15(3): 301-6.

10. Vigneswaran R. Infection and preterm birth: evidence of a common causal relationship with bronchopulmonary dysplasia and cerebral palsy. J Paediatr Child Health 2000;36(4):293-6.

11. Schendel DE, Schuchat A, Thorsen P. Public health issues related to infection in pregnancy and cerebral palsy. Ment Retard Dev Disabil Res Rev 2002;8(1): 39-45.

12. Ovalle A, Martínez MA, Kakarieka ME, Giglio MS, Fuentes A, Gómez R, et al. Hallazgos histopatológicos de la placenta en la rotura prematura de membranas de pretérmino. Relación con la microbiología aislada. Rev Méd Chile 1998;126:911-8.

13. Yoon BH, Romero R, Park JS, Kim CJ, Kim SH, Choi $\mathrm{JH}$, et al. Fetal exposure to an intra-amniotic inflammation and development of cerebral palsy at the age of three years. Am J Obstet Gynecol 2000; 182:675-81.

14. Ovalle A, Martinez MA, Gomez R, Rubio R, Fuentes 
A, Valderrama O. Antibiotic therapy in patients with preterm premature of membranes: a prospective, randomized, placebo controlled study with microbiologic assessment of the amniotic cavity and lower genital tract. Prenat Neonat Med 1997;2:213-22.

15. Kenyon S, Boulvain M, Neilson J. Antibiotics for preterm rupture of membranes. Cochrane Database Syst Rev. The Cochrane Library, Issue 4, 2004.

16. Kenyon S, Taylor DJ, Tarnow-Mordi WO; ORACLE Collaborative Group. ORACLE-antibiotics for preterm prelabour rupture of the membranes: short-term and long-term outcomes. Acta Paediatr Suppl 2002; 91(437):12-5.

17. Crowley P. Corticosteroides con fines profilácticos para el parto prematuro (Revisión Cochrane traducida). En: La Biblioteca de Salud Reproductiva, Número 8, 2005

18. Hofmeyr GJ, Hannah ME. Planned caesarean section for term breech delivery (Cochrane Review) From The Cochrane Library Issue 1, 2006. Chichester, UK: John Wiley, \& Sons

19. Tanir H, Sener T, Tekin N, Aksit A, Ardic N. Preterm premature rupture of membranes and neonatal outcome prior to 34 weeks of gestation. Int J Gynecol Obstet 2003;82:167-72.

20. Juez G, Lucero E, Ventura-Junca P, Tapia JL, Winter A. Crecimiento intrauterino en recién nacidos chilenos de clase media. Rev Chil Pediatr 1989;60:198202.

21. Taylor-Robinson D, Furr PM. Recovery and identification of genital tract Mycoplasmas. Israel $\mathrm{J}$ Med Sci 1981;17:648-53.

22. Claas HC, Melchers WJ, De Bruijn IH, De Graaf M, Van Dijk WC, Lindeman J, et al. Detection of Chlamydia trachomatis in clinical specimens by the polymerase chain reaction. Eur J Clin Microbiol \& Infect Dis 1990;9:864-8.

23. Wang $\mathrm{E}$, Richardson $\mathrm{H}$. A rapid method for detection of group B streptococcal colonization: testing at the bedside. Obstet Gynecol 1990;76:882-5.

24. Odds FC, Bernaerts R. CHROMagar Candida, a new differential isolation medium for presumptive identification of clinically important Candida species. J Clin Microbiol 1994;32:1923-9

25. Villagra E, Martinez MA, Ovalle A. Flora microbiana genital en una población de alto riesgo obstétrico. Rev Chil Obstet Ginecol 1994;59:32-8.

26. Murray PR, Baron EJ, Jorgensen JH, Pfaller MA, Yolken $\mathrm{RH}$ (Eds). Manual of Clinical Microbiology. $7^{\text {th }}$ Ed, 1999 American Society for Microbiology, Washington DC.

27. Nugent R, Krohn M, Hillier S. Reliability of diagnosing bacterial vaginosis is improved by standardized method of Gram stain interpretation. J Clin Microbiol 1991;29:297-301.

28. Romero R, Mazor M, Morrotti R, Avila C, Oyarzún $\mathrm{E}$, Insunza A, et al. Microbial invasion of the amniotic cavity in spontaneous rupture of membranes at term. Am J Obstet Gynecol 1992;166:129-33.
29. McGregor JA, French JI, Jones W, Parker R, Patterson E, Draper D. Association of cervicovaginal infections with increased vaginal fluid phospholipase A2 activity. Am J Obstet Gynecol 1992;167: 1588-94.

30. Capeless E, Damron DP. Cesarean delivery. In: UpToDate, Rose, BD (Ed), UpToDate, Waltham, MA 2005.

31. Gibbs RS, Castillo MS, Rogers PJ. Management of acute chorioamnionitis. Am J Obstet Gynecol 1980; 136:109-13.

32. Papile LA, Burstein J, Burstein R, Koffler H. Incidence and evolution of subependymal and intraventricular hemorrhage: a study of infants with birth weights less than 1,500 gm. J Pediatr 1978;92:529 34.

33. Bernischke K, Kaufman P. Pathology of the human placenta. Chapter: Histopathological Approach to Villous Alteration, 419-434. Chapter: Infectious diseases, 591-659. New York NY: Springer Verlag NY Inc; 2001.

34. Perrin VDK. Pathology of the Placenta. Chapter: Placenta as a Reflection of maternal disease, 57-70. New York, Churchill Livingstone Inc; 1984.

35. Fox H. Pathology of the Placenta. Chapter: Histological Abnormalities of the Placenta, 149-197. Philadelfia, Pa: WB Saunders Co; 1978.

36. Keeling JW. Fetal and Neonatal Pathology. Chapter: Placenta and Umbilical Cord, 45-67. Spriger-Verlag, Berlin Heidelgerg; 1987.

37. Mercer BM, Miodovnik M, Thurnau GR, Goldenberg $\mathrm{RL}$, Das AF, Ramsey RD, et al. Antibiotic therapy for reduction of infant morbidity after preterm premature rupture of the membranes. A randomized controlled trial. National Institute of Child Health and Human Development Maternal-Fetal Medicine Units Network. Jama 1997;278:989-95.

38. Ovalle A, Figueroa J, Gómez R, Martínez MA, Kakarieka $\mathrm{E}$, Fuentes $\mathrm{A}$, et al. Relación entre doppler de la arteria umbilical, invasión microbiana de la cavidad amniótica, funisitis y resultado adverso neonatal en la rotura prematura de membranas de pretérmino. Rev Chil Obstet Ginecol 2005;70(6):375385.

39. Chaiworapongsa T, Romero R, Kim JC, Kim YM, Blackwell SC, Yoon $\mathrm{BH}$, et al. Evidence for fetal involvement in the pathologic process of clinical chorioamnionitis. Am J Obstet Gynecol 2002;186: 1178-82.

40. Pacora P, Chaiworapongsa T, Maymon E, Kim YM, Gomez R, Yoon BH, et al. Funisitis and chorionic vasculitis: the histological counterpart of the fetal inflammatory response syndrome. J Matern Fetal Neonatal Med 2002;11:18-25.

41. Yoon BH, Romero R, Moon JB, Shim SS, Kim M, $\mathrm{Kim} \mathrm{G}$, et al. Clinical significance of intra-amniotic inflammation in patients with preterm labor and intact membranes. Am J Obstet Gynecol 2001;185:11306. 